\title{
Early experience of transcatheter correction of superior sinus venosus atrial septal defect with partial anomalous pulmonary venous drainage
}

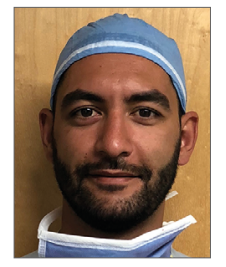

Mounir Riahi ${ }^{1,2}$, MD; Mari Nieves Velasco Forte ${ }^{1,3,4}$, MBBS; Nick Byrne ${ }^{1,5}$, MSc; Anthony Hermuzi ${ }^{1}$, MBBS; Matthew Jones ${ }^{1}, \mathrm{MD}$; Alban-Elouen Baruteau ${ }^{1}, \mathrm{MD}, \mathrm{PhD}$; Israel Valverde ${ }^{1,3,4}, \mathrm{MD}$; Shakeel A. Qureshi ${ }^{1}, \mathrm{MD}$; Eric Rosentha1 ${ }^{1 *}, \mathrm{MD}$

\begin{abstract}
1. Department of Paediatric and Adult Congenital Heart Disease, Evelina London Children's Hospital, Guy's and St Thomas' NHS Foundation Trust, London, United Kingdom; 2. Department of Adult Congenital Heart Disease, St Paul's Hospital, University of British Columbia, Vancouver, Canada; 3. Division of Imaging Sciences and Biomedical Engineering, King's College London, London, United Kingdom; 4 Cardiovascular Pathology Unit, Institute of Biomedicine of Seville, IBIS, Virgen del Rocio University Hospital/CSIC/University of Seville, Seville, Spain; 5. Department of Medical Physics, Guy's and St. Thomas 'NHS Foundation Trust, London, United Kingdom
\end{abstract}

This paper also includes supplementary data published online at: http://www.pcronline.com/eurointervention/141st_issue/155

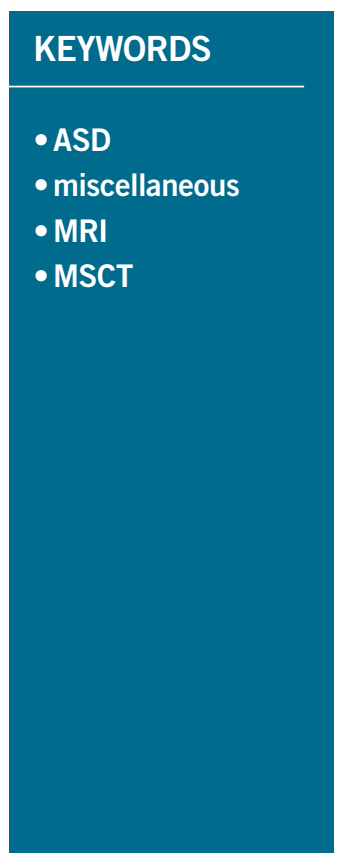

\begin{abstract}
Aims: Superior sinus venosus atrial septal defect (SVASD) is commonly associated with partial anomalous pulmonary venous drainage (PAPVD). We aimed to describe the first series of percutaneous SVASD and PAPVD correction using a two-step simulation for procedural planning.
\end{abstract}

Methods and results: Patients with SVASD and right PAPVD with a clinical indication for correction were selected. They underwent an ex vivo procedural simulation on a 3D-printed model followed by an in vivo simulation using balloon inflation in the targeted stent landing zone. The percutaneous procedure consisted in deploying a 10-zig custom-made covered stent in the SVC-RA junction. Five patients were referred for preprocedural evaluation and were deemed suitable for percutaneous correction. The procedure was successful in all patients with no residual interatrial shunt and successful redirection of the pulmonary venous drainage to the left atrium. At a median clinical follow-up of 8.1 months (2.6-19.8), no adverse events were noted, and all patients showed clinical improvement. During follow-up, transthoracic echocardiography and multidetector cardiac tomography in four patients or invasive angiography in one patient demonstrated a patent SVC stent, and no residual SVASD and unobstructed PV drainage in all patients.

Conclusions: In selected patients using a two-stage simulation strategy, percutaneous correction of SVASD with PAPVD is feasible and safe, and led to favourable short-term outcomes.

\footnotetext{
*Corresponding author: Evelina London Children's Hospital, St Thomas'Hospital, Westminster Bridge Road, London, SE1 7EH, United Kingdom. E-mail: eric.rosenthal@gstt.nhs.uk
} 


\section{Abbreviations}

$\begin{array}{ll}\text { CMR } & \text { cardiac magnetic resonance } \\ \text { LA } & \text { left atrium } \\ \text { LFA } & \text { left femoral artery } \\ \text { MDCT } & \text { multidetector computed tomography } \\ \text { PAPVD } & \text { partial anomalous pulmonary venous drainage } \\ \text { RA } & \text { right atrium } \\ \text { RFV } & \text { right femoral vein } \\ \text { RIJV } & \text { right internal jugular vein } \\ \text { RMPV } & \text { right middle pulmonary vein } \\ \text { RUPV } & \text { right upper pulmonary vein } \\ \text { RV } & \text { right ventricle } \\ \text { SVASD } & \text { superior sinus venosus atrial septal defect } \\ \text { TOE } & \text { transoesophageal echocardiography } \\ \text { TTE } & \text { transthoracic echocardiography }\end{array}$

\section{Introduction}

Superior sinus venosus atrial septal defect (SVASD) is an uncommon form of interatrial communication. Its pathognomonic feature is a right superior vena cava (RSVC) overriding the atrial septum causing an interatrial communication. In about $85 \%$ of cases, the right superior pulmonary venous drainage will connect either at the RSVC-right atrial junction or higher, resulting in partial anomalous pulmonary venous drainage (PAPVD) ${ }^{1,2}$. Surgical correction is considered to be the standard of care and consists in rerouting of the PAPVD to the left atrium (LA) and "septating" the interatrial communication. It has shown favourable long-term results and was, until recently, the only treatment option for this type of lesion ${ }^{3,4}$. A novel transcatheter approach consisting of covering the atrial communication and directing the pulmonary venous flow to the LA by deploying a covered stent in the RSVC to right atrial (RA) junction was first described in 2014 and subsequently in a few isolated case reports ${ }^{5-7}$. In this case series, we aim to describe a reproducible and effective method for evaluation and transcatheter correction of superior sinus venosus atrial septal defects with PAPVD.

Editorials, see page 841 and page 843

\section{Patients and methods \\ PATIENT SELECTION}

Patients with an established diagnosis of SVASD and PAPVD were evaluated by a multidisciplinary Adult Congenital Heart Disease
Team (including at least one congenital cardiothoracic surgeon, two interventional cardiologists, an imaging specialist and an adult congenital cardiologist). From October 2014, those judged to require corrective surgery were considered for transcatheter correction. The indications were symptoms of breathlessness on effort, right heart dilatation on multimodality imaging, Qp/Qs greater than 1.5 by cardiac magnetic resonance (CMR), previous pulmonary emboli or the need for a transvenous pacing lead (Table 1).

\section{PREPROCEDURAL EVALUATION}

All patients had initially been assessed by transthoracic (TTE) and/or transoesophageal (TOE) echocardiography to determine right ventricular (RV) dimensions and function, defect size and position, pulmonary venous drainage, presence and severity of tricuspid regurgitation and right ventricular systolic pressure estimation. CMR or multidetector computed tomography (MDCT) was performed to confirm the diagnosis, evaluate the size of the RV, estimate the $\mathrm{Qp} / \mathrm{Qs}$ ratio and delineate the anatomical relationship of the SVASD and the PAPVD.

Anatomical assessment of the defect and surrounding structures was performed using a multiplanar reformat of the ECG-gated and respiratory navigated three-dimensional balanced steady state free precession (3D bSSFP) acquisition from the CMR (Figure 1) or the MDCT. This allowed segmentation of the anatomy to derive a patient-specific 3D-printed model $^{8}$. All segmentations were carried out on Mimics Medical v18.0 software (Materialise NV, Leuven, Belgium). Models were printed on TangoPlus (ObjetStratasys, Rehovot, Israel), using polyjet technology on an in-house 3D printer (Objet500 Connex1 19M: Objet-Stratasys). The 3D models were printed in a hollow fashion to allow simulation of the procedure and direct inspection of the results (Figure 2, Moving image 1). The dimensions of the stent were estimated from both the cross-sectional images and the model: the length of stent was measured from the site of the landing zone in the SVC to beyond the SVASD to ensure full coverage of the defect. The diameter of the RSVC and right atrium distal to the inferior margin of the defect were used to choose the appropriate balloon size for stent deployment.

\section{PROCEDURAL DETAILS}

All the procedures were performed under general anaesthesia and TOE guidance. TOE with colour Doppler interrogation helped

Table 1. Baseline clinical characteristics.

\begin{tabular}{|c|c|c|c|c|c|c|c|c|}
\hline & $\begin{array}{c}\text { Age } \\
\text { (years) }\end{array}$ & Sex & $\begin{array}{c}\text { BSA } \\
\left(\mathbf{m}^{2}\right)\end{array}$ & Indication & $\begin{array}{c}\text { SVASD size } \\
(\mathbf{m m})\end{array}$ & $\begin{array}{c}\text { RVEDVi } \\
\left(\mathbf{m L} / \mathbf{m}^{2}\right)\end{array}$ & $\begin{array}{c}\text { LSVC } \\
\text { Qp/Qs* }\end{array}$ \\
\hline$\# 1$ & 61 & Male & 2.06 & Significant shunt & 15 & 111 & No & 2.3 \\
\hline$\# 2$ & 66 & Male & 1.86 & Embolic risk & 12 & 91 & No & 1.4 \\
\hline$\# 3$ & 38 & Male & 1.97 & Significant shunt & 27 & 126 & No & 1.7 \\
\hline$\# 4$ & 51 & Female & 1.90 & Significant shunt & 14 & 154 & Yes & 2.6 \\
\hline$\# 5$ & 53 & Female & 1.71 & Significant shunt & 22 & 129 & No & 2.6 \\
\hline
\end{tabular}

${ }^{*} \mathrm{Qp} / \mathrm{Qs}$ was determined using cardiac magnetic resonance. "Need for a transvenous pacemaker lead. ${ }^{\bullet}$ The normal volume for RV end-diastolic volume index is $100 \mathrm{ml} / \mathrm{m}^{2}$. BSA: body surface area; LSVC: left superior vena cava; Qp: pulmonary flow; Qs: systemic flow; RVEDVi: right ventricular end-diastolic volume index; SVASD: superior sinus venosus atrial septal defect 


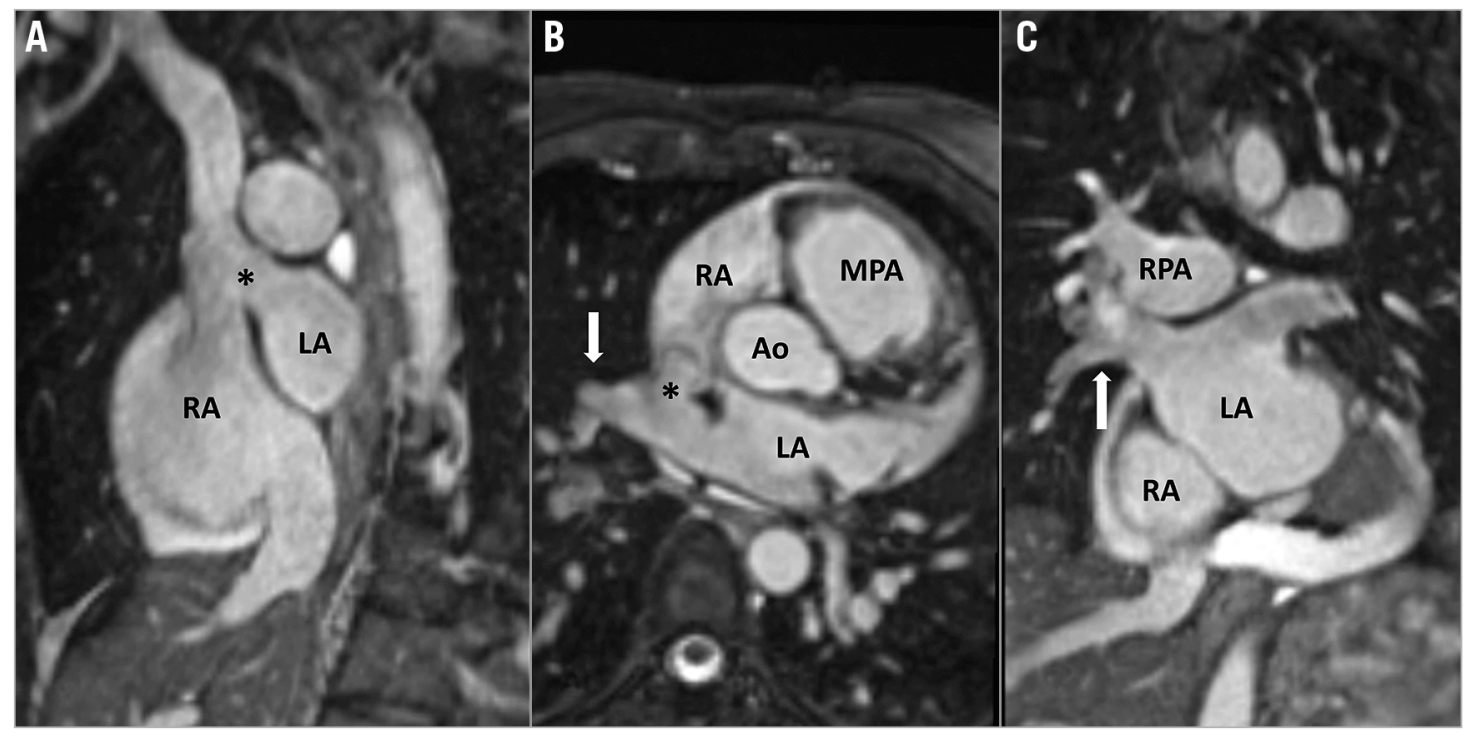

Figure 1. CMR images showing the diagnostic features of the SVASD defect. A) The SVC overrides the atrial septum through the SVASD (*) in a modified sagittal view. B) Right upper and middle pulmonary veins (white arrow) drain into the SVC-RA junction through the SVASD (*). C) Coronal reformat shows pathway of the RUPV and RMPV (white arrow) into the LA. Note: the "cross-road" between SVC, right pulmonary veins, RA and LA cannot be shown in a single plane. Ao: aorta; LA: left atrium; MPA: main pulmonary artery; RA: right atrium; $R M P V$ : right middle pulmonary vein; RPA: right pulmonary artery; RUPV: right upper pulmonary vein; SVASD: sinus venosus atrial septal defect

to assess atrial shunting, monitoring of the pulmonary venous flow and positioning of the stent in the RA. Under ultrasound guidance, access was obtained in the right internal jugular vein (RIJV), the right femoral vein (RFV) and the left femoral artery (LFA). Intravenous antibiotics were administered and intravenous heparin was given throughout the procedure to maintain an activated clotting time (ACT) of over 250 seconds. A $0.035 \% / 260 \mathrm{~cm}$ AMPLATZER $^{\text {TM }}$ wire (St. Jude Medical, St. Paul, MN, USA) was introduced through the RFV, placed in the RSVC and then snared with a Gooseneck snare (Microvena Corp., White Bear Lake, MN, USA) from the RIJV to form a veno-venous guidewire rail. A JR 4 catheter was introduced in the right femoral artery sheath and used to access the right upper pulmonary vein (RUPV) retrogradely via the left ventricle and exchanged for a pigtail angiographic catheter to allow RUPV delineation, serial angiograms and pressure monitoring. An AMPLATZER sizing balloon (St. Jude Medical) was inflated in the RSVC/RA junction. Sequential angiograms from the RIJV sheath and the RUPV
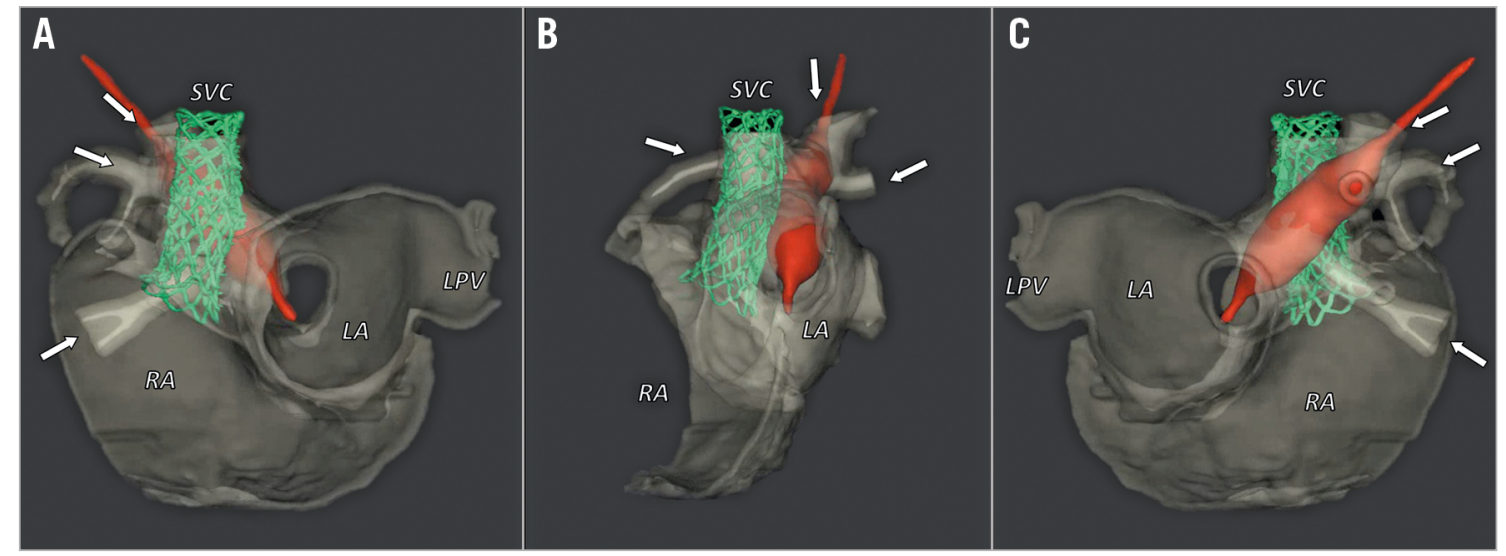

Figure 2. $3 D$ representations of the instrumented, 3D-printed model for patient 4 , rendered from rotational, cone-beam computed tomography scans. A balloon-expanded stent (green) is positioned within the SVC-RA junction to cover the sinus venosus defect. At the same time, a balloon catheter (red) demonstrates continuity between the RUPV and RMPV (arrows) and LA. Arrangement of these structures is shown in anterior-posterior (A), left anterior oblique $(B)$ and posterior-anterior $(C)$ views. See Moving image 1 for a rotating presentation of the simulated procedure. LA: left atrium; LPV: left pulmonary vein; RA: right atrium; SVC: superior vena cava 
catheter were used to confirm RSVC flow occlusion and preserved drainage of the RUPV to the LA (Figure 3A, Figure 3B, Moving image 2, Moving image 3 ). In one patient, 3D rotational angiography confirmed patency of the RUPV. Simultaneous TOE was used to confirm elimination of the interatrial shunt and absence of flow acceleration in the RUPV (Figure 3C, Figure 3D, Moving image 4). A 10-zig custom-made covered Cheatham-Platinum stent (NuMED Inc., Hopkinton, NY, USA) pre-mounted on an appropriately sized BiB balloon (NuMED Inc.) was then introduced through an $18 \mathrm{Fr}$ delivery sheath (Cook Medical, Bloomington, IN, USA) from the RFV and placed in the RSVC-RA junction. Using simultaneous TOE and angiographic
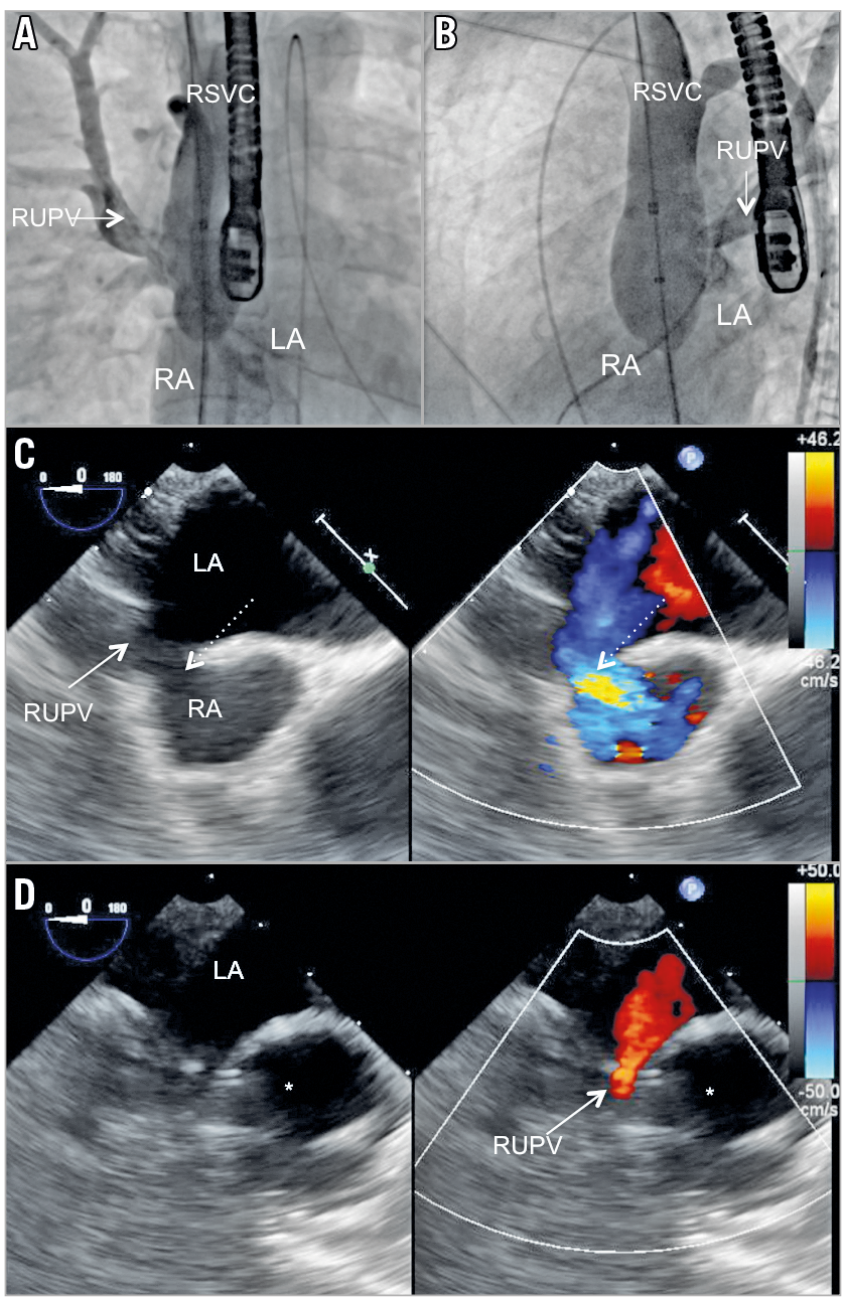

Figure 3. Inflation of a $34 \mathrm{~mm} A M P L A T Z E R$ sizing balloon in the $S V C$ to RA junction covering the SVASD. Injection in the RUPV demonstrates unobstructed RUPV drainage to the LA in A) anteriorposterior view and B) left anterior oblique view. C) TOE short-axis view of the SVC/RA junction showing the SVASD (dashed arrow). D) TOE short-axis view showing patent flow from the RUPV (solid arrow) to the LA during balloon (*) inflation. *indicates the inflated balloon. LA: left atrium; $R A$ : right atrium; $R M P V$ : right middle pulmonary vein; $R S V C$ : right superior vena cava; RUPV: right upper pulmonary vein guidance from the RIJV, the inner and outer balloons were sequentially and progressively inflated to allow precise stent deployment. The caudal end was aimed to sit right below the lower edge of the defect on TOE and the cranial end sufficiently high in the RSVC to allow secure anchoring (Figure 4A-Figure 4C, Moving image 5, Moving image 6). During balloon inflation, tension on the guidewire exiting the RIJV helped to prevent the balloon-stent assembly from migrating into the larger diameter right atrium. The balloon was then deflated, pulled caudally and its outer component re-inflated to flair the RA portion of the stent ensuring complete coverage of the atrial communication (Figure 4D, Moving image 7). Final angiograms from the RSVC and RUPV as well as TOE with Doppler interrogation and bubble shunt study helped to confirm the absence of residual interatrial shunt and flow acceleration in the RUPV (Figure 5A-Figure 5F, Moving image 8-Moving image 11). A z-stitch technique was used for haemostasis after removal of the sheath from the RFV. Procedural success was defined as abolition of interatrial shunt with uncompromised PV drainage and absence of major complication leading to surgical intervention or death.

\section{ETHICAL CONSIDERATIONS}

This novel procedure was first presented to and approved by the Guy's and St Thomas' Clinical Governance Committee following which it was accepted as a "new procedure" by the Guy's and St Thomas' Trust Risk and Quality Assurance Committee. We subsequently obtained authorisation to use custom-made stents on a case-by-case basis through the unlicensed medicinal products programme of the UK Medicines and Healthcare Products Regulatory Agency (MHRA). All patients signed a written informed consent to undergo this novel procedure.

\section{Results}

Between October 2014 and June 2017, five patients were referred for further preprocedural anatomical evaluation to assess suitability for percutaneous SVASD closure and PAPVD flow redirection. Four patients underwent CMR and one patient had an MDCT as part of their assessment.

In all patients, CMR or MDCT was diagnostic of SVASD with PAPVD, involving the right upper pulmonary vein (RUPV) and right middle pulmonary vein (RMPV). Analysis of the cross-sectional imaging and subsequently the three-dimensional models suggested that a transcatheter approach was likely to be successful without compromising pulmonary venous return to the left atrium.

The procedure was performed in these five patients at a median age of 53 years (range: 38-66 years). The clinical indication was symptomatic left-to-right shunt with evidence of right heart enlargement in three patients. In one patient, the haemodynamically significant SVASD had been discovered when she presented with pulmonary embolism. One patient had undergone surgical repair of the SVASD many years previously, but the surgical details were unavailable. During an attempt to place transvenous pacemaker leads when he presented with intermittent complete 


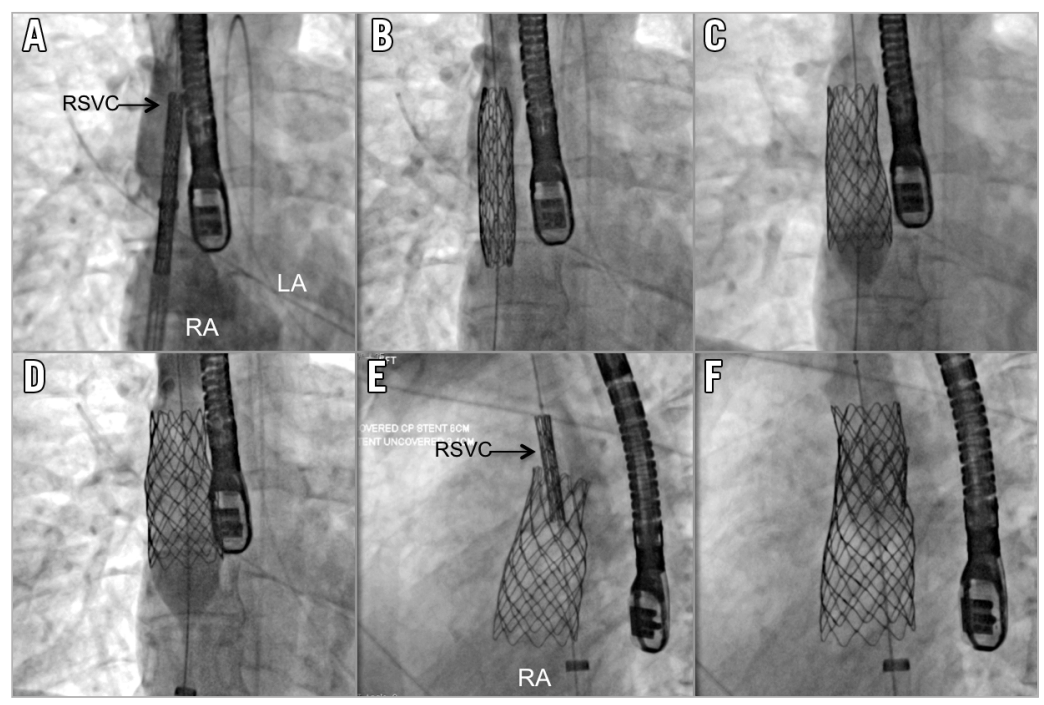

Figure 4. AP view demonstrating progressive deployment of the 10-zig $60 \mathrm{~mm}$ stent on a $26 \mathrm{~mm}$ BiB balloon. A) Unexpanded stent. B) After the initial inflation of the inner balloon, an RSVC injection helped precise positioning of the system in the RSVC to RA junction. C) Full deployment of the outer balloon. D) The balloon was deflated, partially pulled back in the RA and re-inflated to flair the bottom part of the stent. E) \& F) Deployment of a second 8-zig $34 \mathrm{~mm}$ bare metal stent in the upper part of the first stent to provide additional anchoring in left anterior oblique view. LA: left atrium; RA: right atrium; RSVC: right superior vena cava

atrioventricular block, he was discovered to have a residual shunt with anomalous pulmonary vein drainage such that the leads passed into the left atrium and ventricle. All the patients had evidence of right heart enlargement with a median indexed right ventricular end-diastolic volume of $135 \mathrm{~mL} / \mathrm{m}^{2}$ (range: 91-154). The median maximal diameter of the SVASD on 2D TOE was $22 \mathrm{~mm}$ (range: 14-27) with a median Qp/Qs of 2.4 (range: 1.4-2.6) as estimated by CMR. The clinical and anatomical characteristics of the patients are presented in Table $\mathbf{1}$ and Table 2.

The procedure was successful in all five patients with a median procedural time of $190 \mathrm{~min}$ (range: 145-228). In two patients, the operators felt that stent apposition to the RSVC wall might have been insufficient to provide secure anchoring. Therefore, a second 8-zig conventional Cheatham-Platinum bare metal stent
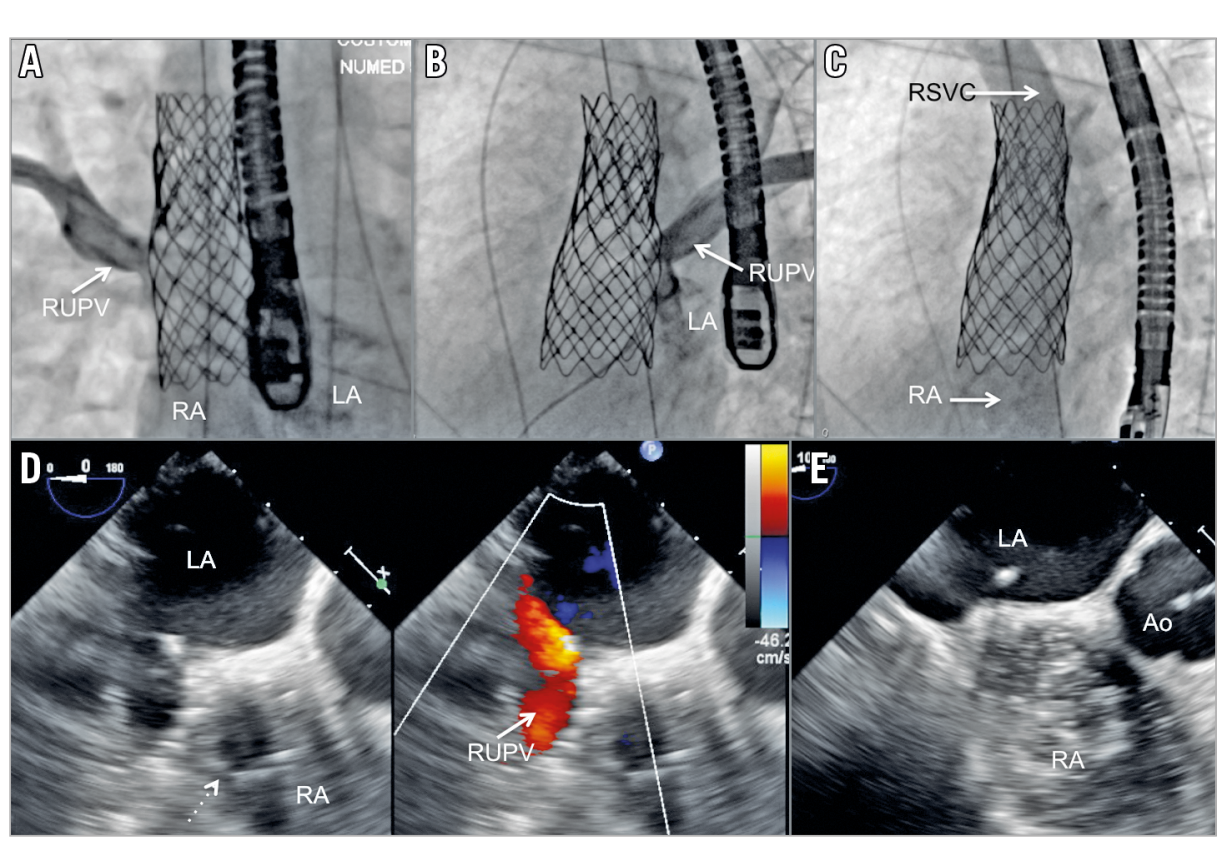

Figure 5. RUPV angiography demonstrating laminar flow of the RUPV to LA, (A: AP view, B: left anterior oblique view). C) RSVC injection delineating unobstructed RSVC to RA pathway without residual interatrial communication. Short-axis TOE view showing laminar RUPV flow after stent (dashed arrow) deployment (D) across the SVASD with laminar pulmonary vein flow (solid arrow). E) Bubble study confirming abolition of the interatrial communication. Ao: aorta; LA: left atrium; RA: right atrium; RUPV: right upper pulmonary vein 
Table 2. Procedural details.

\begin{tabular}{|c|c|c|c|c|c|c|c|c|c|}
\hline & $\begin{array}{c}\text { Balloon } \\
\text { stretched RSVC } \\
\text { segment (mm) }\end{array}$ & $\begin{array}{c}\text { Balloon } \\
\text { stretched RA } \\
\text { segment (mm) }\end{array}$ & $\begin{array}{c}\text { Stent } \\
\text { length } \\
(\mathbf{m m})\end{array}$ & $\begin{array}{c}\text { Balloon } \\
\text { diameter } \\
(\mathbf{m m})\end{array}$ & $\begin{array}{c}\text { RSVC } \\
\text { apposition } \\
\text { length (mm) }\end{array}$ & $\begin{array}{c}\text { Secondary } \\
\text { stent }\end{array}$ & $\begin{array}{c}\text { Procedure } \\
\text { time } \\
\text { (min) }\end{array}$ & $\begin{array}{c}\text { Fluoroscopy } \\
\text { time } \\
\text { (min) }\end{array}$ & $\begin{array}{c}\text { Radiation } \\
\text { dose } \\
\text { (cGy/cm) }\end{array}$ \\
\hline$\# 1$ & 26 & 30 & 60 & 28 & 21 & 0 & 217 & 53 & 25,514 \\
\hline$\# 2$ & 25 & 29 & 50 & 26 & 28 & 0 & 190 & 58 & 10,075 \\
\hline$\# 3$ & 20 & 24 & 60 & 28 & 20 & 0 & 183 & 38 & 12,679 \\
\hline$\# 4$ & 19 & 25 & 60 & 24 & 17 & 1 1 & 228 & 82 & 4,855 \\
\hline$\# 5$ & 18 & 24 & 60 & 26 & 15 & 1 & 145 & 21 & 2,623 \\
\hline
\end{tabular}

" 8-zig bare metal stent $39 \mathrm{~mm}$ length on $16 \mathrm{~mm} \mathrm{BiB}$ balloon. ${ }^{\diamond}$-zig bare metal stent $34 \mathrm{~mm}$ length on $24 \mathrm{~mm}$ BiB balloon. RA: right atrium; RSVC: right superior vena cava

(NuMED Inc.) was deployed in the upper segment of the RSVC below the innominate vein, so as to overlap the cranial portion of the covered stent (Figure 4E, Figure 4F, Moving image 12). There were no procedural complications. The first patient had a femoral vein ooze that settled after application of topical tissue glue.

\section{FOLLOW-UP}

All patients had a TTE, an electrocardiogram and a bi-plane chest $\mathrm{X}$-ray on the following day. They were discharged on aspirin (for six months) and clopidogrel (for two months). A dual-chamber transvenous pacemaker was implanted six weeks after the procedure in the patient in whom pacing had failed prior to the procedure. Routine follow-up included regular TTE and ECGs at three- to six-monthly intervals following the procedure.

At a median follow-up of 8.1 months (range: 2.6-19.8), all patients showed clinical improvement with increased exercise tolerance without evidence of sinus node dysfunction on ECG. Transthoracic echocardiography showed a patent SVC stent without residual shunting or PV obstruction. This was confirmed on MDCT imaging in four patients performed at a median of 53 days (range: 20-502) (Figure 6) and by angiography in the patient undergoing pacemaker implantation.

\section{Discussion}

The introduction and development of a new interventional procedure as an alternative to an established surgical procedure without testing it in a model or animal preparation prior to clinical use raises anxieties and ethical questions. When the defect itself is conventionally considered to be treatable only surgically, it becomes a challenge to the established preconceptions. In 2014, Garg et al successfully closed an SVASD with PAPVD using a covered stent in a patient with bilateral SVCs ${ }^{5}$. This "leap of faith" forced us to re-evaluate our understanding of the anatomy of this defect and led us to develop the technique and modifications of the approach described in this paper. By critically analysing cross-sectional imaging obtained with CMR and MDCT, we realised that selected patients with this anatomy could be treated by an interventional approach with a covered stent. The creation of patient-specific $3 \mathrm{D}$ models confirmed this speculation and allowed us to test the procedure ex vivo, giving us the confidence to proceed to in vivo

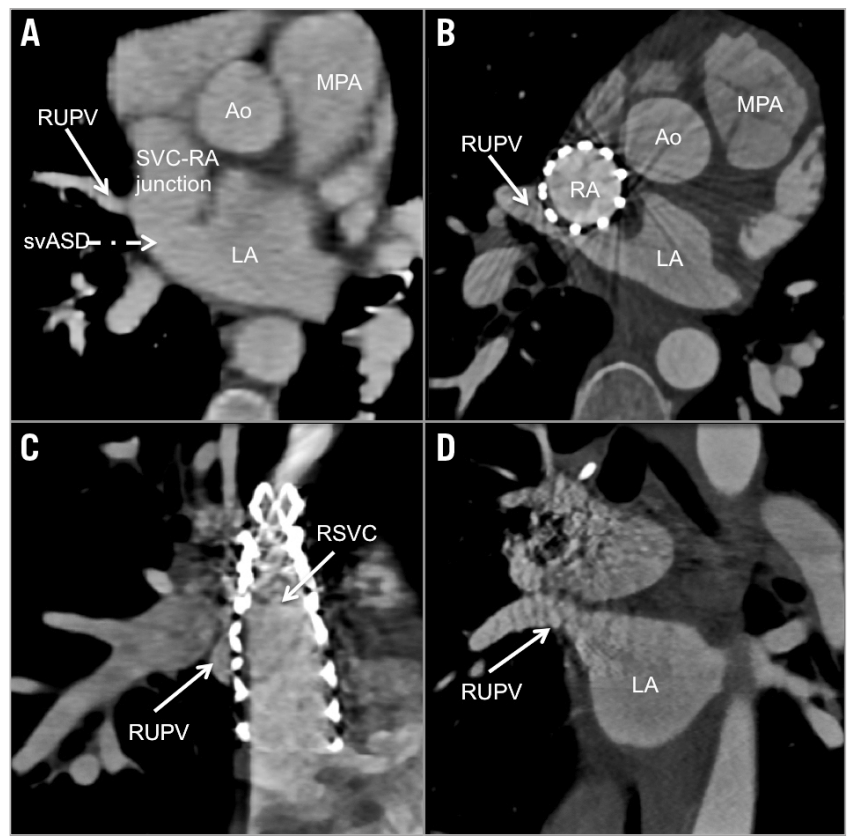

Figure 6. Cross-sectional multiplanar reconstruction of cardiac computed tomography imaging pre-procedure (A) and postprocedure (B-D). A) RUPV drainage at the SVC to RA junction and the SVASD. B) Modified axial view showing a patent RSVC to RA stent covering the SVASD and allowing free RUPV drainage to the LA. C) Sagittal view showing patent RSVC to RA stent obscuring the $R U P V$ channel posterior to the stent. D) The coronal view shows well preserved RUPV to LA drainage. Ao: aorta; LA: left atrium; $M P A$ : main pulmonary artery; $R A$ : right atrium; $R M P V$ : right middle pulmonary vein; $R P A$ : right pulmonary artery; $R S V C$ : right superior vena cava; RUPV: right upper pulmonary vein; SVASD: sinus venosus atrial septal defect

assessment. This was followed by successful implantation of covered stents in five consecutive patients with SVASD and PAPVD. We enrolled our first patient in 2014 but only undertook our first procedure in 2016 after evaluating the 3D models and obtaining institutional and regulatory support.

To understand the procedural concept, it is essential to appreciate the anatomy of this lesion. In a pathological study, Li et al 
showed that the atrial sinus venosus communication is not a "true" septal defect. Rather, it is the consequence of absent infolding of the atrial wall that leads to the RSVC overriding the true atrial septum ${ }^{9}$. The infolding of the atrial wall also serves as the posterior wall of the SVC and anterior wall of the RUPV. In its absence, the RUPV is no longer committed to the left atrium, drains into the SVC-RA junction and overrides the atrial septum. Consequently, anomalous drainage of the RUPV is invariably present and its degree depends on the configuration of the crossroad formed by the SVC mouth, RUPV ostium and right and left atria above the level of the unroofed atrial septum. A covered stent is thus able to separate the common space between the posterior aspect of the RSVC and the anterior portion of the RUPV, simultaneously closing the interatrial communication and redirecting the RUPV flow to the LA.

Given the inherent anatomical variability of this lesion, it is essential to have a thorough understanding of each patient's anatomy when assessing suitability for this procedure. In all the cases, we used multiplanar and 3D reconstruction with either CMR or MDCT to confirm the diagnosis, to size the RSVC and the atrial communication and to evaluate the nature of the RUPV drainage. To estimate the likelihood of successful coverage of the atrial communication without compromising the RUPV drainage to the LA, we performed a two-stage procedural simulation. First, we conducted an ex vivo simulation by deploying a stent in a 3D-printed model based on each patient's MDCT or CMR scans. Coverage of the atrial communication and patency of the RUPV were assessed visually and by using plastic probes or balloon catheters and confirmed by rotational cone-beam CT (DynaCT; Siemens Healthineers, Erlangen, Germany) ${ }^{8}$. A second in vivo simulation was undertaken using balloon inflation in the SVC in order to evaluate the patency of the RUPV during the distention of the native biological tissues. In contrast, Garg et al and Cristal et al only used intraprocedural balloon testing ${ }^{5,6}$, whilst Thakkar et al used 3D printing alone in a single case ${ }^{10}$. In another case report, Gertz et al described procedural planning using CT but it is not clear if intraprocedural balloon testing was performed before the stent implantation? ${ }^{7}$.

During the follow-up, we were able to reassess four patients with MDCT, one with angiography at the time of pacemaker placement and all patients with echocardiography. We noted no stent migration, stent obstruction, PV obstruction or residual shunting. As with the surgical approach, the possibility of sinus node dysfunction is a concern when a stent is implanted in the SVC-RA junction ${ }^{11}$. In our series, all the patients had serial ECGs during the follow-up and none had any sinus node dysfunction, except the patient with prior heart block and previous SVASD surgery.

\section{PROCEDURAL CHALLENGES}

SVC stenting has been widely performed in cases of SVC obstruction and has a low immediate risk of stent misplacement, stent obstruction and bleeding secondary to tissue injury ${ }^{12-14}$.
In relatively large series of SVC stenting for non-malignant obstruction, long-term follow-up showed that freedom from reintervention was $90 \%$ at five years ${ }^{9,11}$. The majority of cases of late stent stenosis were due to intimal hyperplasia, which responded well to repeat angioplasty with drug-eluting balloons ${ }^{15}$. In SVASDs, the stent is placed in a non-obstructed SVC and flared in the RA and so the risk of downward stent migration is potentially higher. We are aware of unpublished reports of acute stent migration in other centres (S.A. Qureshi - personal communication). Our strategy to prevent stent migration is to oversize the diameter of the stent by 3-4 $\mathrm{mm}$ with respect to the SVC size, to ensure sufficient length of apposition of a minimum of $2 \mathrm{~cm}$, and to deploy the stent slowly using the BiB balloon, whilst applying traction to the SVC end of the guidewire rail to counteract the tendency for the stent-balloon assembly to "milk" caudally. In two patients, the mismatch between the diameter of the SVC and the RA at the level of the SVASD raised concerns that "rocking" of the stent might lead to embolisation. We therefore anchored the SVC portion of the stent with an overlapping uncovered stent. The RUPV is accessed in a retrograde fashion from the LA so as not to interfere with RSVC/RA stent placement and avoid catheter trapping. In addition to allowing repeat angiograms to guide the procedure, continuous access to the RUPV ensures the possibility of bail-out balloon dilation or even stenting in the event of unforeseen RUPV obstruction. For stenting the SVC, Garg et al used a balloon-expandable Advanta covered stent (Maquet, Rastatt, Germany) ${ }^{5}$, while Cristal et al used multiple self-expanding covered stents ${ }^{6}$, Gertz et al used overlapping unspecified covered stents ${ }^{7}$ and Thakkar et al used an Endurant $\mathrm{II}^{\circledR}$ Aortic Expansion self-expanding stent (Medtronic, Minneapolis, MN, USA $)^{10}$. We feel that there is likely to be greater control using balloon-expandable stents and therefore had the 10-zig covered stent custom-made together with an appropriate $\mathrm{BiB}$ balloon from NuMED, which allowed a range of diameters to be available.

\section{Limitations}

Surgical correction of sinus venosus ASD has evolved over the years and multiple techniques have been proposed ${ }^{4,16}$. It remains the default therapeutic approach with favourable short- and longterm results. However, in addition to its inherent morbidity and sternotomy scar, surgical repair carries a risk of late SVC stenosis, PV stenosis, residual shunting and sinus node dysfunction requiring pacemaker implantation ${ }^{11,17,18}$. In this context, a transcatheter approach in selected patients appears attractive, although larger experience and longer follow-up are needed before this can be recommended more widely. Our patients were carefully selected, and our technique might not be suitable for a subset of anatomies, such as RUPV drainage that connects superiorly to the SVC/RA junction, because of the risk of obstructing the drainage with the covered stent. In the setting of a relatively hypoplastic RSVC (especially in the presence of an LSVC), overexpansion of the stent immediately caudal to the 
SVC-RA junction could also occlude the RUPV. Currently, we do not advocate this technique in paediatric patients, who are still undergoing significant growth.

\section{Conclusions}

The present study provides early evidence of the safety and reproducibility of transcatheter correction of SVASD with PAPVD in carefully selected patients using a covered stent. The outcomes of a standardised two-stage simulation strategy based on 3D-printed models and invasive balloon interrogation for case selection and procedural planning have been encouraging during short-term follow-up. We believe that this technique could become an alternative to surgical correction in carefully selected patients but longer-term follow-up and larger case series and registries are needed to confirm these results.

\section{Impact on daily practice}

Selected patients with an SVASD and PAPVD in whom surgical correction was previously considered to be the only treatment modality may now have an alternative option of interventional catheterisation correction of their defect without the need for open heart surgery.

\section{Conflict of interest statement}

S. Qureshi is a consultant for NuMED Inc. The other authors have no conflicts of interest to declare.

\section{References}

1. al Zaghal AM, Li J, Anderson RH, Lincoln C, Shore D, Rigby ML. Anatomical criteria for the diagnosis of sinus venosus defects. Heart. 1997;78:298-304.

2. Ferreira Martins JD, Anderson RH. The anatomy of interatrial communications--what does the interventionist need to know? Cardiol Young. 2000;10:464-73.

3. Gaynor JW. Management of sinus venosus defects. Semin Thorac Cardiovasc Surg Pediatr Card Surg Annu. 2006;9:35-9.

4. Stewart RD, Bailliard F, Kelle AM, Backer CL, Young L, Mavroudis C. Evolving surgical strategy for sinus venosus atrial septal defect: effect on sinus node function and late venous obstruction. Ann Thorac Surg. 2007;84:1651-5; discussion 1655.

5. Garg G, Tyagi H, Radha AS. Transcatheter closure of sinus venosus atrial septal defect with anomalous drainage of right upper pulmonary vein into superior vena cava--an innovative technique. Catheter Cardiovasc Interv. 2014;84:473-7.

6. Crystal MA, Vincent JA, Gray WA. The wedding cake solution: A percutaneous correction of a form fruste superior sinus venosus atrial septal defect. Catheter Cardiovasc Interv. 2015;86: 1204-10.

7. Gertz ZM, Strife BJ, Shah PR, Parris K, Grizzard JD. CT angiography for planning of percutaneous closure of a sinus venosus atrial septal defect using a covered stent. J Cardiovasc Comput Tomogr. 2018;12:174-5.
8. Velasco Forte MN, Byrne N, Valverde I, Gomez C, Hermuzi A, Prachasilchai P, Mainzer G, Pushparajah K, Henningson M, Hussain T, Qureshi S, Rosenthal E. Interventional Correction of Sinus Venosus Atrial Septal Defect and Partial Anomalous Pulmonary Venous Drainage: Procedural Planning Using 3D Printed Models. JACC Cardiovasc Imaging. 2018;11:275-8.

9. Li J, al Zaghal AM, Anderson RH. The nature of the superior sinus venosus defect. Clin Anat. 1998;11:349-52.

10. Thakkar AN, Chinnadurai $\mathrm{P}$, Breinholt JP, Lin $\mathrm{CH}$. Transcatheter closure of a sinus venosus atrial septal defect using 3D printing and image fusion guidance. Catheter Cardiovasc Interv. 2018 Jun 13. [Epub ahead of print].

11. Glatz AC, McBride MG, Paridon SM, Cohen MS, Walker SA, Gaynor JW, Tanel RE. Long-term noninvasive arrhythmia assessment after surgical repair of sinus venosus atrial septal defect. Congenit Heart Dis. 2010;5:141-8.

12. Tzifa A, Marshall AC, McElhinney DB, Lock JE, Geggel RL. Endovascular treatment for superior vena cava occlusion or obstruction in a pediatric and young adult population: a 22-year experience. J Am Coll Cardiol. 2007;49:1003-9.

13. Rizvi AZ, Kalra M, Bjarnason H, Bower TC, Schleck C, Gloviczki P. Benign superior vena cava syndrome: stenting is now the first line of treatment. J Vasc Surg. 2008;47:372-80.

14. Sheikh MA, Fernandez BB, Gray BH, Graham LM, Carman TL. Endovascular stenting of nonmalignant superior vena cava syndrome. Catheter Cardiovasc Interv. 2005;65:405-11.

15. Breault S, Doenz F, Jouannic AM, Qanadli SD. Percutaneous endovascular management of chronic superior vena cava syndrome of benign causes: long-term follow-up. Eur Radiol. 2017;27:97-104.

16. Ling Y, Gan C, Sandeep B, An Q. Modified Warden procedure for partial anomalous pulmonary venous return to the superior vena cava. J Card Surg. 2016;31:631-4.

17. Alsoufi B, Cai S, Van Arsdell GS, Williams WG, Caldarone CA, Coles JG. Outcomes after surgical treatment of children with partial anomalous pulmonary venous connection. Ann Thorac Surg. 2007;84:2020-6.

18. Attenhofer Jost CH, Connolly HM, Danielson GK, Bailey KR, Schaff HV, Shen WK, Warnes CA, Seward JB, Puga FJ, Tajik AJ. Sinus venosus atrial septal defect: long-term postoperative outcome for 115 patients. Circulation. 2005;112:1953-8.

\section{Supplementary data}

Moving image 1.3D printed model with simulated intervention.

Moving image 2. Right upper pulmonary vein angiography with balloon sizing of SVC - AP view.

Moving image 3. Right upper pulmonary vein angiography with balloon sizing of SVC - left oblique view.

Moving image 4. Transoesophageal echocardiography during balloon sizing of SVC.

Moving image 5. Right internal jugular angiography during inflation of inner balloon.

Moving image 6. Fluoroscopy during outer balloon inflation. 
Moving image 7. Right internal jugular angiography while flaring stent in right atrium.

Moving image 8. Right internal jugular angiography after stent implantation.

Moving image 9. Right upper pulmonary vein angiography after stent implantation.

Moving image 10. Transoesophageal echocardiography after stent implantation - colour Doppler.
Moving image 11. Transoesophageal echocardiography after stent implantation - contrast injection.

Moving image 12. Deployment of anchoring stent in SVC.

The supplementary data are published online at: http://www.pcronline.com/

eurointervention/141st issue/155 